\title{
Groove Sens(e)ations - mit und über Empfindungen forschen
}

Sebastian Matthias

\section{Zusammen grooven}

Groove aus der Perspektive der Zuhörer*innen rhythmischer Musik beschreibt meist das Bedürfnis, mit zu wippen, zu bouncen oder zu tanzen. Groove reißt mit, strukturiert Bewegung im Zusammenspiel mit dem Gehörten scheinbar von selbst und erzeugt so das Gefühl einer gewissen Leichtigkeit, frei von vorherigen Absprachen oder Skripts. Unter Jazzmusiker*innen ist Groove ein in Bewegung empfundenes ästhetisches Ideal ${ }^{1}$ und dient als Richtschnur für eine gelingende Interaktion: Im bewegten Körper spüren Musiker*innen, ob sie zusammen »hot « spielen (Widmeier 2004: 1). Dabei empfinden nicht nur Musizierende diese Groove sens(e)ations. Clubbesucher*innen strömen allwöchentlich auf Elektro-Partys, um an der mit Groove umschriebenen kollektiven Rhythmuserfahrung teilzuhaben. Die sensationelle Körpererfahrung beim Höhepunkt einer Clubnacht oder bei Raves versuchen sie dabei, mit Begriffen wie »vibe« (Fikentscher 2000: 80), »collective energy« (2000: 88) oder eben Groove zu bezeichnen. Das wiederholte Empfinden von Groove markiert für die Tanzenden in ihrem Körpererleben die entscheidenden Momente einer gelungenen Party.

Für die Mehrheit der Besucher*innen erscheint die von ihnen empfundene Energie aus der Musik und dem DJ-Set zu entspringen. So laut und unentrinnbar die Bässe brummen, so glaubwürdig erscheint die Dominanz

1 Das Konzept von musikalischem Groove im Jazz zielt auf eine performative, produktive Verknüpfung unterschiedlicher Rhythmen, die mit einer »motionalen« (Butterfield 2010: 173) Qualität als »ästhetische[m] Ideal« (Monson 1996: 201) verbunden ist. 
der Musik über den Tanz. Erst die zaghaften Versuche, eine Tanzfläche zu eröffnen, oder die innere Schwere, die aufkommt, wenn eine Gruppe den Dancefloor verlässt, rückt die Choreografie der Körper entschiedener in das pulsierende Zentrum der Groove-Erfahrung: Wenn es leichter erscheint, gemeinsam zu tanzen als allein, dann deshalb, weil erst das Sich-Organisieren in der Versammlung von Körperbewegungen diesen Groove erzeugt. Tänzerischer Groove als kinästhetisch empfundenes Ideal und Richtschnur organisiert und erleichtert im Clubtanz kollektiv die Tanzbewegungen. Die Körperempfindung als qualitatives Messinstrument steht hierbei im Zentrum des Groove-Phänomens. Die Erforschung von Groove wird so zu einem paradigmatischen Fall dafür, wie Körperempfindungen sinnhaft wirken können und im Medium von Körperbewegungen eine bestimmte Art von Wissen generieren. Groove eröffnet eine Alternative zu zeichengestützter Kommunikation und macht einsichtig, wie performative kunst-gestützte Forschung das Wissen über dessen Organisation fassbar machen kann.

\section{Empfindung als Messinstrument}

Im Folgenden werde ich meine künstlerisch-wissenschaftliche Forschung zu Bewegungsinteraktionen im Clubtanz nachzeichnen und einen methodischen Zugang vorstellen, wie ephemere Phänomene, die auf subjektiven Empfindungen beruhen, wissenschaftlich gefasst werden können. Meine Leitfrage dabei wird sein: Welche Forschungsverfahren können genutzt werden, um in affektives und sensorisches Erleben eingebettetes Wissen zu verstehen, darzustellen und für größere semiotische, ästhetische und soziale Fragestellungen fruchtbar zu machen?

Eine Groove-Dynamik in der Feldforschung als eindeutiges Beispiel herauszulösen, um deren Organisationsmodi zu verstehen, ist eine analytische Herausforderung. Es gibt nicht »den« einen Groove-Moment, sondern nur einzelne Groove-Dynamiken und deren Bewegungsinteraktionen, die sich zudem nicht durch reine formale Beschreibung, sondern erst unter Einbeziehung persönlich empfundener Erfahrungen eingrenzen lassen. Der Moment, in dem sich eine Clubbesucherin im Groove fühlt, muss nicht auch für ihren Nachbarn groovy sein. Zudem sind Fülle, Vielfalt und Feinsinnigkeit der Aktivitäten auf der Tanzfläche im Bild oder Video nur schwer oder höchstens unvollständig sichtbar zu machen. Der improvisatorische Cha- 
rakter von Clubtanz entzieht sich einer vollständigen Erfassung, etwa durch Video-gestützte Analyse. Um diese Schwierigkeiten zu überwinden, wurde ein partizipatives, durch künstlerische Verfahren gestütztes Forschungsdesign entwickelt, innerhalb dessen qualitative Studien im Feld und Labor, Bewegungsexperimente im Studio und in Clubs durchgeführt wurden. Die subjektiven Empfindungen der Teilnehmer*innen, festgehalten in Bewegungsprotokollen und Fragebögen, waren die Grundlage für die Analyse der hier vorgestellten Forschung (Matthias 2018). Die daran anschließenden Modelle von Bewegungsorganisationen wurden auf sechs choreografische Arbeiten der groove space-Serie übertragen, um die choreografischen Strukturen aus dem Club in ihrer Übersetzung in den performativen Raum zu testen. Die groove space-Serie enthält fünf Choreografien, die als freie Projekte oder Auftragsarbeiten in Berlin, Zürich, Freiburg, Jakarta und Düsseldorf/ Tokyo in Kollaboration mit wechselnden Künstler*innengruppen erarbeitet wurden. ${ }^{2}$ Die künstlerischen Beispiele überführen Bewegungsstrukturen bzw. Organisationsprinzipien aus dem Clubtanz in choreografische Operationen und testen so deren Übertragbarkeit und weitere Funktionsweisen. Mit der Anwendung in einem neuen Feld wird ihr Verständnis geschärft, um dieses wiederum für die Feldforschung fruchtbar zu machen und deren Prämissen neu hinterfragen zu können. In einem rekursiven Prozess informieren sich im Forschungsablauf Kunst und tanzwissenschaftliche Forschung gegenseitig. Der Transfer vom Club zur Kunst bleibt ein artifizieller Schritt, doch zeigt sich in der Übertragbarkeit ein eigenständiges Bewegungsphänomen, das nicht nur auf den Clubtanz begrenzt ist.

Aus dem umfangreicheren Forschungsdesign möchte ich im Folgenden drei experimentelle Setups vorstellen. Hierbei handelt es sich um verschiedene performative Zugänge, die nicht in dieser Vereinzelung und Reihenfolge das Forschungsdesign bestimmt haben ${ }^{3}$, aber anhand derer ich die Ergebnisse der Grooveforschung hier in einem erklärungs- und kunstgestützten Analysemodell vorstellen möchte. Die Übungen sind keine bloße Illustration, sondern eine Übersetzung der Problemstellung in ein andersartiges Forschungssetting, in dem die Körpererfahrung als Grundlage des folgenden Argumentes dient. Außerhalb der Komplexität des Clubs können so das ge-

\footnotetext{
2 Als weiterführendes Projekt habe ich Yuropa (2018, Bremen) im Abgleich mit zeitgenössischen nigerianischen Musik- und Tanzpraktiken choreografiert.

3 Für ein ausführliches Forschungsdesign meiner Forschung siehe Matthias (2018).
} 
wissermaßen destillierte Phänomen neu oder zum ersten Mal erspürt und einzelne Bewegungsinteraktionen in einen neuen Sinnzusammenhang gestellt werden. Die Empfindungen, die in den folgenden experimentellen Setups erlebt werden, beforschen und befragen so selbst das Groove-Phänomen. Die performativen Setups generieren Körpererleben als Orte der Wissensproduktion, wobei dem Körpererleben im Sinne der Forschungsergebnisse die gleiche Autorität wie analytischen und theoretischen Verfahren zukommt. Mit dem Körper als Messinstrument im Zentrum versuchen sich die Experimente, dem Groove-Phänomen performativ zu nähern und herauszuarbeiten, warum Tanzende es aus choreografischer Perspektive als einfacher empfinden können, zusammen zu tanzen als alleine.

\section{Setup 1: Kontrastierung - Empfinden impulsgebender Differenz}

Zehn Teilnehmende stellen sich mit dem Rücken nach innen in einen Kreis. Jeweils ein*e Teilnehmer*in erhält die Aufgabe, in der Mitte der Aufstellung kontinuierlich zu eingespielter Musik zu tanzen. Die Teilnehmenden begeben sich einzeln nacheinander in die Tänzer*innenrolle, um die Wirkungsweisen von Anweisungen an die umstehenden Teilnehmenden aufden eigenen Tanz mit geöffneten und geschlossenen Augen zu erleben. Die im Kreis stehenden Teilnehmenden erhalten folgende Anweisungen:

Anweisung »1 «-jeder tanzt, wie er will;

Anweisung »2«-jeder bounct mit den Knien und dem Oberkörper;

Anweisung $3 \ll-$ alle stehen still.

In Setup $1^{4}$ wird gefragt, ob und wie Bewegungen im Umfeld eines Tanzenden dessen Bewegungsempfinden verändern und den Bewegungsaufwand verringern können. Anweisungen 1 und 2 stellen voneinander verschiedene dynamische Bewegungsumfelder her, während die Anweisung 3 eine statische Umgebung hervorbringt, die den Bewegungsaufwand $\mathrm{zu}$ hemmen scheint. Durch die Differenz und den abrupten Übergang von einem Umfeld zum anderen kann der Einfluss der Bewegungsumgebungen auf den eigenen Tanz miteinander verglichen werden. In seiner formalen Struktur kann das Setup vergleichbare Aussagen der Teilnehmenden über deren Performativität gene-

4 Dieses Setup kann unter https://vimeo.com/248352215 nachvollzogen werden. 
rieren. Generell beschreiben Teilnehmende aber eine Erleichterung der eigenen Bewegung, wenn im Umfeld alle Teilnehmenden mittanzen. ${ }^{5}$ Wie kann dieser Einfluss gefasst werden? Was bedeutet zunächst die erste Anweisung: zur Musik tanzen? Welches Repertoire rufen die Tanzenden dabei auf?

Zu EDM (Electronic Dance Music) oder Clubmusik synchronisieren die Tanzenden ihren Körper mit einer inneren Erwartungshaltung zu den sich überlagernden Rhythmuspatterns. Sie folgen ganz oder partiell im gegenwärtigen Moment der musikalischen Struktur und einem gefühlten strukturierenden Verlauf in einer improvisatorischen Weise. Diese Synchronisation fällt mit einer bekannten House-Musik leicht und kommt im Groove fast von allein. Die Teilnehmenden tanzen einfach los, müssen nichts planen und greifen auf ihre persönliche Cluberfahrung zurück. Wenn im Groove der Kopf beginnt, zum Beat mit zu wippen, sprechen Jessica Phillips-Silver et al. von "akustischem Entrainment« (Phillips-Silver/Aktipis/Bryant, 2010: 2). Es ist eine Grundlage der menschlichen Zeitwahrnehmung, indem ein externer periodischer Stimulus mit einer motorischen Synchronisierung verknüpft wird. Diese mitreißende Synchronisierung kann entlang unterschiedlicher sensorischer Modalitäten erfolgen und dient zur Wahrnehmungsverarbeitung zeitlicher Prozesse. Einer chronologischen Denkweise fällt es schwer, parallele Intervalle zu denken und gleichzeitig wahrzunehmen. Der Körper hilft sich selbst mit der Verknüpfung, um zwei Zeitspannen einmal spüren und einmal hören zu können, um diese miteinander abzugleichen. In virtuosen Improvisationen greifen Tänzer*innen dabei auf ein ihnen bekanntes und/oder teilweise gerade beobachtetes Bewegungsrepertoire zurück. Mit Hilfe von akustischem Entrainment werden Bewegungsmotive in ihrem Verlauf in die musikalische Matrix der Beats gesetzt. Geordnet durch ein gefühltes flüssiges Zusammenspiel der Bewegungsmotive und der Auswahl der mehrschichtigen Rhythmuspatterns werden Tanzbewegungen immer wieder variiert und angepasst. Im Clubtanz kommt es dabei nicht auf die korrekte Setzung der Beine im Raum an, sondern auf wellenartige Übertragungen, die den Körper zwischen den impulsgebenden Setzungen zur musikalischen Matrix hin zu autonomen und daraus resultierenden Bewegungen in Schwingung verset-

5 Die Probanden der vorliegenden Versuchsreihe machen diese Aussage (Matthias 2018: $82 \mathrm{ff}$ ) und in der Wiederholung dieses Experiments in verschiedenen Kontexten und Gruppen wurden ähnliche Beschreibungen gemacht. Doch vor allem im Kontext von Bühnentänzer“innen wurde beschrieben, dass die stille Umgebung besser für ihren Tanz war. 
zen. Diederichsen beschreibt für EDM, dass betonte rhythmische Strukturen sich "von innen anders« (Diederichsen 2014: 217) anfühlen. Nach Diederichsen kann durch eine veränderte Haltung oder innere Einstellung zur Musik eine andere, autonome, öffnende Funktion der rhythmischen Spur wahrnehmbar werden (2014: 217). Wie entsteht dieses innere Sich-Anfühlen aus Entrainment, dem verkoppelten Sich-Angleichen an die rhythmische Matrix?

In Anweisung 2 kann im Bounce dieses innere Sich-Anfühlen in einer reduzierten Form nachvollzogen und getestet werden. Bounce als Bewegungsmotiv wird hier als eine Grundlage für den gemeinsamen Tanz gesehen. Die wellenartigen Übertragungen sind im Bounce isoliert und in dieser vereinfachten Struktur abstrahiert, damit ihre Funktion für den Körper als Messinstrument verständlich und überprüfbar werden kann. Bounce wird verstanden als eine kleinstmögliche Einheit von Clubtanz, die zum Beispiel grundlegend für das beliebte Step-Touch-Motiv (Matthias 2018) gesehen werden kann: Bounce ist eine Form eines körperlichen Rhythmuspatterns, das den Körper des Wippenden in eine kontinuierliche Schwingung bringt. Durch die elastische Spannung in den Muskeln, die das Gewicht immer wieder hochschnellen lässt, bleiben beim Bouncen die Beine in einer Bewegungsschleife, die den Körper in eine konstante Bewegungsqualität des Fallens und Wiederansteigens bringt. Eine leichte Beugung der Arme und der Wirbelsäule leitet den Bewegungsimpuls an den restlichen Körper weiter und bringt ihn so in eine bouncende Bewegungsqualität. Die anatomischen Konsequenzen der Impulsübertragung bringen den ganzen Körper ohne direkte Steuerung in Bewegung. Durch die Nervenenden in den Gelenken und der Haut in der Propriozeption (O'Shaughnessy 1995) drängt die kinästhetische - körperlich empfundene - Raum- und Körpererfahrung in die Aufmerksamkeit der Tanzenden. Aufmerksamkeit wird in der rezessiven Propriozeption durch Reizveränderung herbeigeführt. Die Empfindung der Position von Körperteilen im Raum, wird - wenn auch immer präsent - entweder durch gezielte Aufmerksamkeitssteuerung oder durch Bewegung bewusst, sonst versinkt der propriozeptische Reiz im Filter der Aufmerksamkeit. Lassen sich Tanzende vom Rhythmus eines House- oder Dub-Step-Beats mitreißen und verfallen im Entrainment in eine bounceartige Bewegung, empfinden die Rezipienten im Körper das Beatintervall von House und Dub-Step durch unterschiedliche Übertragungswellen völlig verschieden. Das innere Sich-Anfühlen des Groove in der Musik bekommt eine somatische oder kinästhetische Evidenz durch motorische Synchroni- 
sierung. Das Groove-Feld im Bounce in Anweisung 2 kann so als ein kleinster gemeinsamer Nenner zwischen improvisiertem Tanz und dem Bewegungsumfeld der homogenen Gruppe gesehen werden, dessen Performanz für die Teilnehmenden in der Mitte des Kreises getestet werden kann.

Im Gegensatz dazu stehen die stillen Körper in Anweisung 3. In meinem persönlichen körperlichen Erleben fühlt sich die Umgebung von Anweisung 3 an wie eine Schwere, die mich zu Boden zieht. Wird wieder gemeinsam weitergetanzt, gibt es mir in der Mitte einen besonderen Schub und erzeugt eine neue Leichtigkeit der Bewegung. Bei Experimenten im Club hat sich gezeigt: Wenn Probanden sich still in die Mitte gestellt haben, drehten sich die anderen tanzenden Clubbesucher weg, um die stillen Körper aus ihrem Umfeld auszuschließen (Matthias 2018).

Der Bounce durch Entrainment eröffnet ein Denkmodell, wie Musik zu einer groove sens(e)ation im Körper werden kann. In welcher Beziehung stehen jedoch die Bewegungen von den Tanzenden in der Mitte zum Umfeld? Wie kann ein homogenes Bewegungsfeld im Bounce mit der freien Improvisation eine Beziehung eingehen, die eine veränderte Bewegungserfahrung nach sich zieht? Welche Interaktionen sind für die groove sens(e)ations zwischen Tanzenden verantwortlich? Um dies praktisch nachzuvollziehen, wird ein Setup vorgestellt, das durch eine stärkere Vereinfachung und Abstraktion diese Bewegungsbeziehung körperlich nachvollziehbar macht.

\section{Setup 2: Vereinfachung - Empfinden zerstreuter Kommunikation}

Mehr als zehn Teilnehmende platzieren sich alleinstehend im Raum und folgen erst mit geschlossenen, dann geöffneten Augen choreografischen Anweisungen, die durch eine eingespielte Musik mit Beatstruktur unterstützt wird.

1. Stehe gerade mit dem Gewicht auf beiden Füßen. Folge mit der Aufmerksamkeit den Mikrobewegungen, die den Körper ähnlich wie in Steve Paxtons »small dance" aufrecht halten (Paxton 1977: 11). Folge diesen Bewegungsimpulsen. Erkenne, wie das Gewicht sich organisiert. Erkenne, wie es zu einer Suspension der Richtungsänderung kommt. Erkenne, wie das Gewicht mit der Bewegung zurückgleitet. Erkenne, wie sich dies in seiner eigenen Zeitlichkeit organisiert.

2. Erweitere die Gewichtsübertragung in den Raum, indem du sie von einem Bein aufdas andere gleiten lässt. 
3. Erkenne ein spezifisches Gleiten in der Gewichtsübertragung, dass als eine qualitative Einheit gesehen werden kann. Stelle aktiv das Gleiten im Fluss von einer Bewegungsrichtung zur anderen her. Als Grundstruktur dieser Improvisation behalte dieses Gefühl in den folgenden Anweisungen bei.

4. Bewege das Gleiten nicht nur von Seite zu Seite, sondern auch vor und zurück. In der Suspension der Richtungsänderung vollziehe eine leichte Drehung, die die Seitbewegung in eine Vor-und Rückbewegung bringt.

5. Beginne, frei die Richtungen zu wählen und einen Fluss in der Richtungsänderung zu generieren.

6. Folge diesem Fluss und öffne die Augen. Wohin fällt dein Blick bei der Richtungsänderung aus der Gewichtsübertragung?

7. Erweitere den Bewegungsweg, indem du die Gleitintervalle durch Schritte verlängerst. Lass dich von dem gleitenden Gefühl leiten.

8. Durchlaufe gleitend den Raum ohne den Rhythmus der Gewichtsübertragung zu brechen.

9. Was passiert, wenn ich jemandem begegne? Wie reagiert mein Körper? Weiche ich der Nähe aus? Gleiche ich mich an die empfangenen Richtungsimpulse an?

10. Verringere den genutzten Raum, so dass alle Teilnehmenden enger beisammen sind. So eng, dass ich bei jeder Richtungsänderung jemandem begegne.

11. Wenn ich meine Gewichtsübertragung beibehalten will, wie organisiere ich mich dann mit der Gruppe?

12. Wie organisiere ich meinen Bewegungsweg? Wie entscheidet sich die neue Richtung? Verhilft oder hemmt ein gleicher Rhythmus den ungebrochenen Fluss?

Die Anweisungen in Setup 2 bringen ein choreografisches System hervor, das in chorus/groove space (2015) entwickelt wurde. Dies stellt eine Übertragung und Vereinfachung der Interaktion von Clubtanzbewegungen dar, die zum Beispiel in schwungvollen Seitschritten nachzuweisen sind (Matthias 2018). In diesem choreografischen System kann die körperliche Erfahrung von Synchronisierungen und De-Synchronisierungen getestet und nachvollzogen werden. Anstatt seinen Weg durch den Raum zu planen, sich einen Weg $\mathrm{zu}$ bahnen, bewegen sich Tanzende von Moment zu Moment und richten sich nach den Bewegungen der anderen aus, gleichen sich an oder drehen sich in eine andere Richtung, um sich auszuweichen. Dieses Sich-Angleichen fühlt sich einfach an und muss nicht geprobt oder gezielt choreografiert werden, sondern organisiert sich in der Gruppe von selbst. Es entsteht ein Gefühl von Fluss, der die Bewegenden immer weiter durch den Raum gleiten lässt. 
Aus der Begegnung entsteht ein ausweichender Bewegungsimpuls, der die Bewegenden auf eine neue Bewegungsbahn bringt. Wenn diese wiederum auf einen nächsten Tanzenden trifft, entsteht eine weitere Neuausrichtung, die sich mit dem neuen Bewegungsimpuls regeneriert und damit Tanzende immer weiter gleiten lässt. Die Reduzierung der Abstände verstärkt diese fortwährenden Begegnungen. Tanzende koordinieren nicht die zeitlichen Anfangspunkte der Gewichtsübertragung, sondern gleichen sich mit der angewiesenen gleitenden Qualität allmählich an die anderen Tanzenden an. Diese Angleichung wird durch Antizipation in der Bewegungswahrnehmung erleichtert, da in der Wahrnehmung von Bewegung die Kontinuität der Bewegung mitgedacht wird (Verfaillie/Daems 2002).

Die Kopplung zwischen antizipierender Bewegungswahrnehmung und motorischem System im intuitiven Ausweichen bei einer Begegnung kann mit akustischem Entrainment zusammengedacht werden. Die Tanzenden werden, wenn auch langsam, umgelenkt und wie bei grooviger Musik leicht mitgerissen und in eine neue Richtung gebracht. Das Mitreißen wird verstärkt, wenn verschiedene Richtungen aufeinander einwirken. Hier wird Liz Waterhouse's et al. (2014) Unterscheidung gefolgt und die Angleichung mit dem qualitativen Verlauf von Bewegung als Alignment verstanden. Alignment sehen Waterhouse et al. als "automatic and strategic processes of communication" (Waterhouse/Watts/Bläsing 2014: 14). Im Alignment muss anders als beim Nachtanzen einer fixierten Bewegungssequenz nicht die gesamte Wegstrecke abgewartet und erkannt werden. Bewegung ist hier nicht gedacht als retrospektive Konstruktion eines linearen Wegs, sondern als dynamische Einheit (Massumi 2002: 2f.). Indem sich Tanzende in Form von Alignments immer wieder neu an Teilverläufe angleichen, bewegen sie sich in einem zerstreuten Umfeld, das eine antizipierende Wirkung auslöst. Im Zulassen der Wirkungsweisen von bewegten Umfeldern kann dieses Angleichen wie Musik mitreißend sein und deshalb soll diese Bewegungsbeziehung als eine Form visuellen Entrainments verstanden werden, da sie sich Verarbeitungsprozessen bedient, die visuelle Stimuli mit motorischer Synchronisierung verknüpft.

Wie hier vereinfacht und abstrahiert körperlich erfahrbar, so gibt das kurzweilige Angleichen der qualitativen Verläufe der eigenen Bewegungswahrnehmung einen kurzen Schub und reißt die Tanzenden in ihrer mit dem Umfeld verknüpften motorischen Bewegung mit. Das Bewegungsfeld generiert mitreißenden choreografischen Groove. Das visuelle Entrainment ist nicht wie ein Beat auf eine metrisch zirkuläre Struktur angewiesen, son- 
dern auf eine sich dynamisch angleichende Qualität. Teilverläufe von unterschiedlichen Referenzpersonen mit korrelierendem qualitativem Verlauf verdichten und verknüpfen sich zu einem Feld, wobei es keinen Unterschied zu machen scheint, in welchem Moment der Gewichtsübertragung zwei Tanzende zusammentreffen oder auf welchen Teil des Körpers der Blick fällt. Ein Arm oder Kopf, der den Bewegungsmodus ausführt, wirkt in der Angleichung ebenso affektiv wie ein Blick auf den Rumpf. Wird die Angleichung mit dem qualitativen Verlauf von Bewegung als Alignment gedacht, setzt der plurale Kommunikationsprozess an einem qualitativen zirkulären Bewegungsmodus an, der mit Bewegungsmodi wie Schub, Bounce oder Fall verglichen werden kann. Die spezifischen Relationen zwischen Kraftaufwand, Bewegungsweg und Zeitstruktur konstituieren den Bewegungsmodus wie hier im Gleiten oder im Club im Bounce oder Schwung.

So kann die Beziehung in Anweisung 2 von Setup 1 zwischen homogenem Bounce und freier Improvisation verstanden werden: Die Bewegungsqualitäten in der freien Improvisation ähneln stilistisch oft bounceartigen Bewegungen, z.B., wenn bei Hüpfern zum Beat der Körper mitwippt. Diese bouceartige Qualität der Teilverläufe können dann mit dem homogenen getanzten Bounce in der Anweisung verknüpft werden (Matthias 2018). Die kurzen visuellen Kontaktaufnahmen im wechselnden Blick zwischen Referenzpersonen wirken wie ein kollektiver Bezugsrahmen, auf den sich Tanzende in ihrem Referenzfeld immer wieder stützen. Hierbei haben tänzerische Bewegungen, die einen mitreißen und sich verstärken, keine kausal zwingende Wirkungsweise, denen Clubbesucher*innen ausgeliefert sind. Tanzende müssen nicht mit ihrem Kopf zu einem Beat mitwippen, verspüren jedoch eine Lust, wenn sie beginnen, ihren Körper zur Musik oder dem Bewegungsumfeld mitzubewegen. Hört die Musik auf oder stehen die anderen Teilnehmenden bei Setup I (Anweisung 3) still, ist der einzelne Tänzer oder die einzelne Tänzerin immer noch in der Lage, weiterzutanzen, wenn es seine oder ihre Intention ist. Die Tanzenden werden nicht wie eine Marionette von der Musik oder vom Tanz »automatisch « bewegt.

Setup 2 macht zudem sichtbar, wie sich die Choreografie der Gruppe auf ein körperliches Bewegungsverständnis stützt. Das erlebte Ausweichen generiert Synergien und vernetzt die kollektive Bewegung sinnvoll. Ähnlich dem akustischen Entrainment kann man diese Beziehung auch als körperliche Verarbeitung visueller Eindrücke verstehen, die in der Gruppe einen Schwarmeffekt hervorbringen. Ein »Schwarmeffekt [entsteht] aus der Or- 
ganisierung einer kleinmaschigen Vernetzung jeweils Einiger mit Einigen, wobei die Netzsegmente ineinandergreifen und so den Informationsfluss ausweiten. (van Eikels 2013: 204) Die lokalen Angleichungen der Bewegungsrichtung, die durch die Verständigung auf bestimmte Bewegungsqualitäten sichtbar werden, etablieren zwischen den Tanzenden einen Schwarmeffekt, wobei ein Schwarm hier als eine Art »strategische Matrix kollektiven Agierens« (2013: 204) verstanden werden muss. Zwischen den Tanzenden ergibt sich eine kleinmaschige Vernetzung, die durch Angleichungen vollzogen wird. Die Synergien des kollektiven Agierens basieren auf einer zerstreuten Informationsübertragung in pluraler Kommunikation.

Die Informationsübertragung stellt kollektiv eine kinästhetische Evidenz her, die sich in Setup 2 als gelingender gefühlter Fluss erleben lässt. Dabei ist der einzelne qualitative Verlauf des Gleitens die Einheit, die den gefühlten Bewegungseindruck des wahrgenommenen Umfeldes strukturiert. Der Groove beim gefühlten Fluss, der in Setup 2 erfahren wird, sollte als gut vernetzte Kommunikation verstanden werden. Wird dieser gefühlte Fluss mit dem Körper erfahren, so wird die körperlich erlebte Organisation als sinnvoll wahrgenommen. Die Groove-Erfahrung kann jedoch nicht nur auf eine Vernetzung von Bewegung reduziert werden. Das Gefühl von Groove, indem sich der Körper als eine unstillbare Quelle der Bewegungsgenerierung manifestiert und die Clubnacht im Körper als Ort des Wissens ihren Sinn findet, benötigt noch ein weiteres Element, das in Setup 3 nachvollzogen werden kann.

\section{Setup 3: Zuspitzung - Empfinden gestaltender Variabilität}

In einem engen Kreis stehen sich 6 Personen gegenüber. Eine eingespielte Beat-Musik passt mit ihrem Beatintervall genau in die folgenden Bewegungsanweisungen:

1. Aufden Beat schiebe dein Gewicht mit einem Schritt weg von deinem Zentrum nach vorne. Kurz bevor du nach vorne fallen würdest, ziehe dein Gewicht zurück auf das hintere Bein und bringe dein Gewicht mit Schwung aufdas andere Bein. Der Schwung wird umgelenkt, so dass das Gewicht wieder nach vorne geschoben werden kann. Wiederhole den Wechsel zwischen Schub und seinem Zurückziehen, damit ein Bewegungsloop entsteht.

2. Kommt mit der Gruppe und der Musik in einen gemeinsamen Rhythmus. 
3. Werdet schwungvoller, damit die Grenze der Richtungsänderung weiter in den Raum verschoben werden kann. Versucht, den Körperschwerpunkt immer etwas weiter nach außerhalb zu schieben.

4. Werdet noch schwungvoller.

5. Gleicht euch an die Bewegungen der anderen Tanzenden an.

6. Löst den Kreis auf und versucht, über Betonungen im Schub in die Nähe von Anderen zu kommen.

7. Kann der Schub der anderen Bewegungen meine Bewegungen verstärken? Können andere Bewegungen in dieses Netz eingebaut werden?

8. Lassen sich Variationen daraus ableiten? Kann der Schwung ornamentiert werden? Wie wird der kollektive Schwung zu einer Improvisation genutzt?

Setup 3 stellt ein choreografisches System dar, in dem ausprobiert werden kann, wie eine kraftvolle Umgebung die eigene Bewegung verstärken kann. Es wurde in Zusammenhang mit der Produktion Yuropa 2018 entwickelt. Durch den Schwung der Partner*innen wird die Grenze überreizt, in der die Füße am Boden bleiben und ein kleiner Sprung daraus wird. Der Schwung kann genauso in einer Drehung abgelenkt werden, so dass eine neue Ausrichtung zur Gruppe entsteht. In dieser neuen Position müssen andere Körperteile aktiviert und umgelenkt werden, um den gemeinsamen Schwung aufrechtzuerhalten. In Setup 3 kann ausprobiert werden, wie das GrooveFeld nicht nur die Bewegung erleichtert, sondern die Gruppe der Tanzenden die Konsequenz der gemeinsam getanzten Bewegungsqualität im Körper potenziert. Die Impulsübertragung wird kollektiv verstärkt, so dass sich die resultierende Bewegung stärker ausdifferenziert und erweitert. Folgt ein Tanzender den Konsequenzen seiner Impulse in seinem Körper und spürt den anatomischen Gegebenheiten nach, bringen diese ihn in neue Haltungsrelationen seiner Körperteile, die er nicht vorher geplant hatte. Der Bewegungsimpuls leitet sich zwar von der Musik und dem gemeinsamen Bewegungsmotiv $a b$, aber in der Konsequenz der kollektiv verstärkten Impulsübertragung kann er eine Eigenständigkeit erlangen, welche nicht akustischem Entrainment folgt. Ähnlich einem Ornament entwickelt die Bewegung eine erweiterte Materialität im Vollzug, die die Beschaffenheit der Ursprungsbewegung ausdifferenziert. Durch die Materialität des Körpers entsteht in der Verstärkung eine Erweiterung oder ein »Mehr« seiner eigenen Präsenz und Performanz. Dieser performative Überschuss muss nicht bewusst zur Musik getanzt werden. Improvisierende können sich so von 
ihrem eigenen Tun überraschen lassen, also zum »Zuschauer und Zuhörer ihrer Handlung und ihrer Konsequenzen" (Bormann/Brandstetter/Matzke 2010: 13) werden. Durch den Rückbezug auf die Materialität des Körpers und der Fähigkeit, sich vom Körper überraschen lassen zu können, ergibt sich eine Distanz zum eigenen Tun, das mit der Koordinierung zur Musik immer wieder abgeglichen und in das flüssige Zusammenspiel gesetzt werden muss. Wie im Clubtanz vollzieht sich in Setup 3 ein doppelt verknüpfter Abgleichungsprozess, zum einen mit der Musik und zum anderen mit dem eigenen Körper. Die Musik erleichtert durch Entrainment und die Bewegungskonsequenzen die Improvisation.

Im Club sind Groove-Felder nicht so homogen wie hier im Kontext von Setup 3. Kurzzeitig kann dieser choreografische Groove als solcher auch im Club aufscheinen, doch sind im Clubkontext überlagerte Felder die Regel, in denen sich Angleichungsprozesse an verschiedene Bewegungsqualitäten plural überkreuzen. Das flüssige Wechselspiel zwischen einzelnen Motiven in der Improvisation bietet eine Vielzahl von Anknüpfungspunkten mit verschiedenen Bewegungsumfeldern. An dieser Stelle divergieren die künstlerischen Beispiele mit den Prinzipien der Clubs. Denn im Club ruft eine Ausdehnung des Raumwegs in eine Drehung zum Beispiel eine Körperkoordination hervor, die mit ihrer neuen Qualität neue Bewegungsmotive zur Musik anstößt und der Improvisation neue Wendungen gibt. Die Tanzenden brauchen dafür die Freiheit, ihre Bewegungsqualität wechseln zu können. Zur Herstellung des folgenden Prinzips benötigt man die Intention aller, gemeinsam zu tanzen, und die Bereitschaft, die Bewegungsmotive im eigenen Tanz zu verändern.

Wird eine Improvisation durch das Groove-Feld verstärkt, damit ein choreografischer Überschuss entsteht, dann trifft im Club durch die vielen gemeinsam und zerstreut Tanzenden diese »neue« ausdifferenzierte Bewegungsqualität der resultierenden Bewegung auf ein überlagertes korrelierendes Groove-Feld. Mit dieser neuen Umgebung findet wieder eine mitreißende Synchronisierung statt. Das Ornament verfängt sich nicht wie in Setup 1 wieder mit dem Schwung, sondern mit einem anderen Groove-Feld, das eine neue andere Qualität besitzt. In der Dynamik des gemeinsamen Tanzens wird in der Verstärkung des Groove-Feldes eine Konsequenz im Körper der Tanzenden hergestellt, die einer bewussten Choreografie vorgelagert ist. Durch das Verfangen von Ornamenten in überlagerten Groove-Feldern kann sich eine Endlosschleife stabilisieren, in der die noch nicht bewusst getanzte Bewegung durch die kollektive Verstärkung in die Improvisation drängt. Es 
entsteht ein Gefühl von Groove, in dem sich der Tanzende immer wieder in neuen Bewegungsmotiven wiederfindet, ohne diese vorausplanen zu müssen. In der sich fortschreibenden Improvisation können Tanzende freudig den sich verändernden Bewegungsmodi nachspüren. In der gleichzeitigen Übertragung von Bewegungen wird diejenige verstärkt, die bereits schon in der impulsgebenden Bewegung angelegt war und die durch den eigenen Tanz auch erst in einem anderen Akteur produktiv werden konnte.

Tänzerischer Groove ist also eine zutiefst intime körperliche Erfahrung, die jedoch auf die Teilnahme von Mittanzenden angewiesen ist. So könnte bei der Groove-Erfahrung von einem Modus ausgegangen werden, bei dem sich drei kontinuierliche Informationsflüsse in der Wahrnehmung und Handlung überkreuzen und gegenseitig bestärken: Die akustische Verarbeitung der zyklischen Musik, z.B. mit den Beinen, die verstärkende Synchronisierung des Tanzes im Umfeld und das Nachspüren des eigenen Körpers, der in der flüssigen Ausarbeitung der Bewegungsmotive die Wahrnehmung immer wieder vor neue Haltungsrelationen und Körpererfahrungen stellt.

\section{Tänzerischer Groove als körperliches Emergenzphänomen}

Tanzende im Club haben im Kommunikationsprozess keine festgelegte Position, von der eine abgeschlossene Information ausgeht, um bei einem Empfänger anzukommen. Die Synchronisierungsmechanismen werden zeitgleich wirksam, so dass Tanzende sich im Modus des tänzerischen Grooves gleichzeitig in den Positionen des Neugenerierenden, des Unterstützenden und des Mitgerissenen befinden. Für Groove müssen alle Positionen besetzt werden, so dass für den pluralen Kommunikationsprozess eine kritische Anzahl an Teilnehmenden notwendig wird. Tänzerischer Groove als körperliches Emergenzphänomen erzeugt Sinn nicht als Bedeutung, sondern als sinnvolle Organisation. Er generiert ein systeminhärentes Wissen um die Dynamik zwischen den Tanzenden. Der Körper ist dabei das qualitative Messinstrument, das misst, ob und wie stark die drei oben genannten Informationsflüsse produktiv ineinandergreifen. Der tänzerische Groove umkreist einen Zustand zwischen einem kollektiv verstärkten und einem individuell differenzierten kontinuierlichen Bewegtsein. In diesem Sinne ist Groove im Tanz kein Motiv, keine Qualität wie in der Musik (Pfleiderer 2006), sondern ein tänzerischer Modus - ein Modus der Wahrnehmung und 
Verarbeitung von flüssigen Übertragungen, Übersetzung und Transport von Informationen mit multiplen Partnern und Feldern.

Mit ihrer spezifischen kinästhetischen Erfahrungsdimension steigert kollektives Improvisieren im Clubtanz die Musikerfahrung zu einem subjektiven Körpererleben, welches durch das Kollektiv erst ermöglicht wird. Nur die Versammlung kann diese Körpererfahrung ermöglichen. Sens(e) ations im Club sind nicht nur durch Berührung oder introspektiven Zugang $\mathrm{zu}$ erfahren, sondern entstehen auch durch visuelle Stimuli im Umgang mit Umgebungen. Nach diesen Überlegungen und erspürten Erfahrungen in den Clubs und in den experimentellen Setups möchte ich herausstellen, dass Tanz und Bewegungen nicht nur Sinn und Sinnhaftigkeit darstellen oder dokumentieren, sondern auf der Ebene der kinästhetischen Erfahrung eine Sinnhaftigkeit in der Organisation selbst herstellen. Diese Sinnhaftigkeit birgt eine andere Logik der Kommunikation, die auf die Produktion von Bedeutung in der Übertragungsdynamik verweist und nicht auf einer bildhaften oder sprachlichen Ebene agiert. Groove sens(e)ations werden in der Übertragungsdynamik der tänzerischen Kommunikation konfiguriert und generieren ihren Sinn systeminhärent. Aus meiner Erfahrung mit partizipativer künstlerischer Forschung kann ein solches empfundenes Wissen, damit es in theoretischen und ästhetischen Diskursen sein spezifisches epistemisches Potenzial entfalten kann, nur durch ineinandergreifende Forschungs-Setups in Ko-Forschung mit anderen Tanzenden dargestellt, dokumentiert und nutzbar gemacht werden.

\section{Literatur}

Bormann, Hans-Friedrich/Brandstetter, Gabriele/Matzke, Annemarie (2010): Improvisieren: eine Eröffnung, in: Hans-Friedrich Bormann/Gabriele Brandstetter/Annemarie Matzke (Hg.), Improvisieren: Paradoxien des Unvorhersehbaren. Kunst-Medien-Praxis, Bielefeld:transcript, S. 7-19. Butterfield, Matthew (2010): Participatory Discrepancies and the Perception of Beats in Jazz, in: Music Perception. An Interdisciplinary Journal, Jg. 27 Nr. 3, S. 157-175.

Diederichsen, Diedrich (2014): Über Pop-Musik, Köln: Kiepenheuer \&Witsch. Fikentscher, Kai (2000): »You Better Work!« Underground Dance Music in New York City, Hanover, NH: Wesleyan University Press. 
Massumi, Brian (2002): Parables of the Virtual: Movement, Affect, Sensation, Durham: Duke University Press.

Matthias, Sebastian (2018): Gefühlter Groove - Kollektivität zwischen Dancefloor und Bühne, Bielefeld: transcript.

Monson, Ingrid (1996): Saying Something - Jazz Improvisation and Interaction, Chicago: University of Chicago Press.

O'Shaughnessy, Brian (1995): Proprioception and the Body Image, in: Jose Luis Bermudez/Anthony J. Marcel/Naomi M. Eilan (Hg.), The Body and the Self, Cambridge: MIT Press, S. 175-204.

Paxton, Steve (1977): Interview mit Elizabeth Zimmer, in: Contact Quarterly, Jg. 3 Nr. 1, S. 11.

Pfleiderer, Martin (2006): Rhythmus: psychologische, theoretische und stilanalytische Aspekte populärer Musik, Bielefeld: transcript.

Phillips-Silver, Jessica/Aktipis, C. Athena/Bryant, Gregory A. (2010): The Ecology of Entrainment: Foundations of Coordinated Rhythmic Movement, in: Music Perception, Jg. 28 Nr. 1, S. 3-14.

van Eikels, Kai (2013): Die Kunst des Kollektiven: Performance zwischen Theater, Politik und Sozio-Ökonomie, München: Wilhelm Fink.

Waterhouse, Elizabeth/Watts, Riley/Bläsing, Bettina E. (2014): Doing Duo a Case Study of Entrainment in William Forsythe's Choreography >Duo<, in: Frontiers in Human Neuroscience, Jg. 8, Art. 812, o. S.

Verfaillie, Karl/Daems, Anja (2002): Representing and anticipating human actions in vision, in: Visual Cognition, Jg. 9 Nr. 1-2, S. 217-232.

Widmaier, Tobias (2004): Groove, in: Albrecht Riethmüller (Hg.), Handbuch der musikalischen Terminologie, Stuttgart: Franz Steiner.

Im Zeitraum der Dissertation sind in Kollaboration mit unterschiedlichen Künstler*innenteams verschiedene Choreografien entstanden:

synekism/groovespace (2014): Berlin.

maneuvers/groove space (2014): Zürich.

chorus/groovespace (2015): Freiburg i.Br..

volution/groovespace (2015): Jakarta.

$x /$ groove space (2016): Düsseldorf/Tokyo.

Yuropa (2018): Bremen. 